\title{
Polymer - Solvent Relation: Swelling and Fibre Morphology
}

\author{
Adeakin O.A.S ${ }^{1}$, Popoola V.A ${ }^{2}$ andAjekwene K.K. ${ }^{3}$ \\ ${ }^{1,3}$ Department Of Polymer And Textile Technology, Yaba College Of Technology, Yaba, Lagos, Nigeria. \\ ${ }^{2}$ Department Of Chemistry, Federal University Of Technology, Akure, Nigeria.
}

\begin{abstract}
The concept of solubility parameters was applied in the study of modification achievable on polyethylene terephthalate. The amount of solvent retained and the level of swelling achievable was studied after the polymer was pretreated in ten organic solvents for 30,60,120,300, 900 seconds at $30^{\circ} \mathrm{C}$ and $50^{\circ} \mathrm{C}$ respectively. The amount of solvent retained was determined by weighing using a 4 digit meter balance and recorded as mol/ $\mathrm{kg}$. The results retained were plotted as a function of time and temperature. Optimum retention of the solvents were recorded within 120 seconds of pretreatment after which a desorption was obtained for all the solvents. Benzyl alcohol has a solubility parameter of 12.1 similar to that of the aromatic component of the polymer which showed a higher retention of the solvents at higher temperatures of pretreatment. This is believed to have a swelling effect on the polymer after drying.
\end{abstract}

Keywords: Polyethylene Terephthalate, Solvent Pre-treatment, Swelling, Fiber Morphology

\section{Introduction}

Commercial importance of polyethylene terephthalate is gaining popularity as one of the most versatile engineering polymers due to its excellent mechanical properties. It has found wide application in textile, medical, food packaging, microelectronics and solid state devices. Works by Gokhale et al 1957, showed that chemical treatment of PET with different substances like strong acids and bases as well as certain solvents bring about changes in the fine structure. Much concern have been raised particularly since an increase in its application in food and beverages packaging industry are likely to affect human life. It might not be unusual to find that a polyester container finds application in water, oil and food packaging after its original use have been concluded. A study of the effect of solvent application on polyester is carried out to determine the amount of solvent retained, its structural modification and swelling achievable.

Moore and Sheldon 1961 observed that the nature, polarity type, solubility parameter and molecular size of a solvent were the governing factors for both the equilibrium swelling and crystallinity. They further suggested that the non-polar hexane and carbon tetrachloride did not induce crystallinity. The polarisable aromatic hydrocarbons e.g. benzene did so. They therefore concluded that swelling might be considered as a measure of the interactions between the polymer and the solvent. Bendak 1991 identified that polyester fibres treated with benzyl alcohol, benzene and chloroform increased in swelling as the treatment temperature increase.

\section{Materials and Methods}

The polyester fibre used for this study was obtained from a local mill Lagos. Ten organic solvent were obtained at analar grade (dimethylformamide, acetone, chloroform, benzaldehyde, n- hexane, nitrobenzene, cychlorohexanone, benzyi alcohol, m-crezol and dimethyl-o-phthate). A wrap reel was used to prepare a uniform sample size and kept in a dessicator ready for solvent treatment. A 3-neck solvent treatment flask was used to applied at $30^{\circ} \mathrm{C}$ and $50^{\circ} \mathrm{C}$ respectively using a regulated heating mantle positioned at the bottom of the flask.

\section{Results/ Discussions}

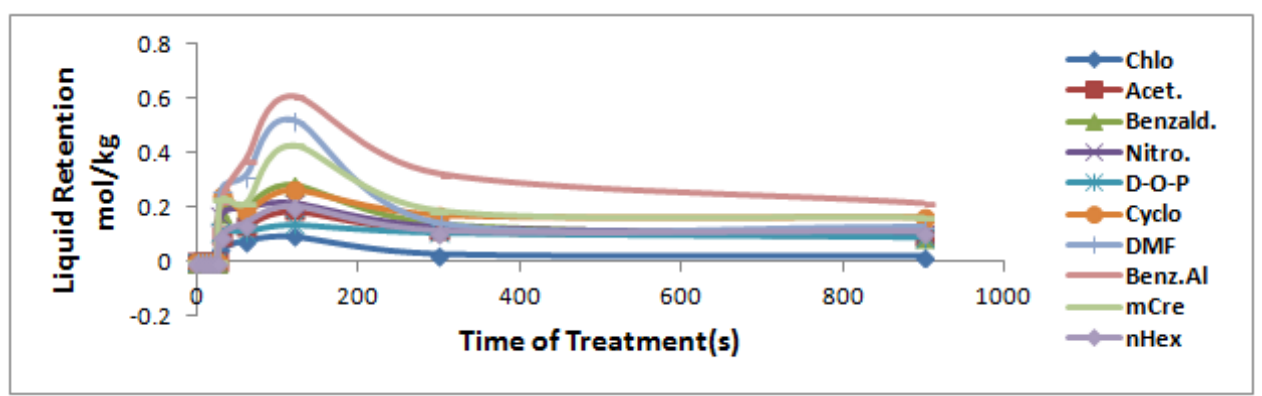

Figure 1 shows Amount of Liquid Retained against Time (mol/kg) at $30^{\circ} \mathrm{C}$ 


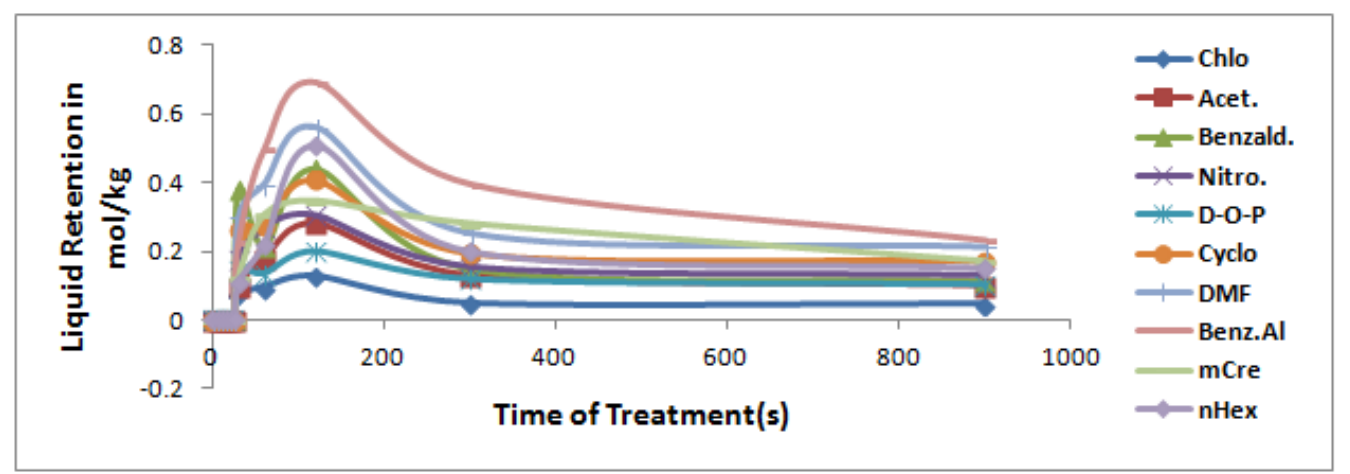

Figure 1 shows Amount of Liquid Retained against Time $(\mathrm{mol} / \mathrm{kg})$ at $50^{\circ} \mathrm{C}$

The treatment of the polyester with the various solvents leads to a rapid intake in the liquids as observed in figures 1 and 2. According to Arrhenius theory the energy supplied for the solvents to diffuse into the polymer structure increased substantially with increase in the experimental temperature $\left({ }^{\circ} \mathrm{C}\right)$ from $30^{\circ} \mathrm{C}$ to $50^{\circ} \mathrm{C}$ and so is the increase in the segmental mobility within the polymer structure. This is manifested in higher peak values for polymer studies at $50^{\circ} \mathrm{C}$ peaking about $/$ around $0.7 \times 10^{-3} \mathrm{moles} / \mathrm{kg}$ as opposed to the average peak value of about $6.2 \times 10^{-4} \mathrm{moles} / \mathrm{kg}$ for polymers at $30^{\circ} \mathrm{C}$.

The rapid rise in the liquid absorption was followed by a sharp drop after about 120seconds in the level of liquids. The loss of the solvents is associated with change going on within the polymer. As liquids are introduced into the polymer structure at higher temperature it provides the necessary chemical energy for release of stresses within chains. This is manifested in the uncoiling and disentanglement of the molecular chains. If these were to be the only morphological changes taking place, a normal kinetic curve depicting a rise in the liquid retention to a point of equilibrium would have been obtained.

\section{Conclusions}

As a result of pretreatment in solvents, polymer solvent contact is established to lead to the polymer retaining some degree / level of solvent. The amount of solvent retained is also found to influence the structure and hence inherent properties of the polymer. The level of retention and the properties as a result of modification is believed to be a function of the solubility parameters. Solvents with solubility parameter on the nearest that of the polymer exhibiting greater influenceon the properties in terms of time and as well as a function of temperature. Benzyl alcohol with a solubility parameter of 12.1 similar to that of polymer hence shows the greater influence on the polymer after pretreatment.

\section{References}

[1]. A. Bendak and S. M. El-Marsafi, Effect of Chemical Modifications on Polyester Fibre. Journal of Islamic Academy of Sciences 4:4, 275-284, 1991.

[2]. M. K. Gokhale, L. Peters and C. B. Stevens, Dyeing Hydrophobic Fibres in Solutions of Solvents. Society of Dyers and Colorists Symposium, 1957.

[3]. W. R. Moore and R. P. Sheldon, The Crystallization in Polyethylene Terephthalate by Organic Liquids. Polymer 2, p 315-321, 1961.

[4]. T. Wakida, Y. Tomihara, I. Aoki and N. Kuroki, Effect of Medium on the Structure of Nylon 6 and Polyester Fibre Treated by Microwave Heating, 1975. 